Annales Geophysicae (2002) 20: 957-965 (c) European Geophysical Society 2002

\title{
The relativistic electron response in the outer radiation belt during magnetic storms
}

\author{
R. H. A. Iles ${ }^{1}$, A. N. Fazakerley $^{1}$, A. D. Johnstone ${ }^{1}$, N. P. Meredith ${ }^{1}$, and P. Bühler ${ }^{2}$ \\ ${ }^{1}$ Mullard Space Science Laboratory, University College London, Holmbury St. Mary, Dorking, Surrey, UK \\ ${ }^{2}$ Paul Sherrer Institut, Laboratory for Astrophysics, 5232 Villigen PSI, Switzerland
}

Received: 1 October 2001 - Revised: 6 February 2002 - Accepted: 5 March 2002

\begin{abstract}
The relativistic electron response in the outer radiation belt during magnetic storms has been studied in relation to solar wind and geomagnetic parameters during the first six months of 1995, a period in which there were a number of recurrent fast solar wind streams. The relativistic electron population was measured by instruments on board the two microsatellites, STRV-1a and STRV-1b, which traversed the radiation belt four times per day from $L \sim 1$ out to $L \sim 7$ on highly elliptical, near-equatorial orbits. Variations in the $E>750 \mathrm{keV}$ and $E>1 \mathrm{MeV}$ electrons during the main phase and recovery phase of 17 magnetic storms have been compared with the solar wind speed, interplanetary magnetic field $z$-component, $B_{z}$, the solar wind dynamic pressure and $D_{s t}{ }^{*}$. Three different types of electron responses are identified, with outcomes that strongly depend on the solar wind speed and interplanetary magnetic field orientation during the magnetic storm recovery phase. Observations also confirm that the L-shell, at which the peak enhancement in the electron count rate occurs has a dependence on $D_{s t}$ *
\end{abstract}

Key words. Magnetospheric physics (energetic particles, trapped; storms and substorms) - Space plasma physics (charged particle motion and accelerations)

\section{Introduction}

The dynamics of the relativistic electron population $(E>$ $0.5 \mathrm{MeV})$ in the Earth's outer radiation belt $(3<L<7)$ during magnetic storms is not well understood (e.g. Li and Temerin, 2001). Reeves (1998) examined the relationship between the relativistic electron population and magnetic storms (defined by $\mathrm{D}_{\mathrm{st}}$ ) during the interval 1992-5, using a detector on the geosynchronous satellite 1989-046. He found that although every relativistic electron flux enhancement coincided with a magnetic storm, not every storm led to an increase in the relativistic electron flux. In this study, the correlation between the maximum electron flux during a magnetic

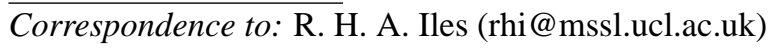

storm and the minimum $D_{s t}$ was also found to be fairly poor. Thus, a magnetic storm appears to be a necessary, though not a sufficient, condition for relativistic electron flux enhancements at geostationary orbit. Some additional condition relating to the magnetosphere or the solar wind must also be involved. The relationship between relativistic electron flux enhancements and increases in the solar wind speed was first reported by Williams and Smith (1965) and Williams (1966) and later by Paulikas and Blake (1979). In a further study, Blake et al. (1997) correlated changes in the relativistic electron population with the up-stream solar wind conditions and found that "a large relativistic electron enhancement depends upon a substantial solar wind speed increase associated with a precursor solar wind density enhancement, and, in particular, upon a southward turning of the interplanetary magnetic field". Here, we perform a detailed study of 17 magnetic storms in which we relate the relativistic electron response to the solar wind conditions throughout each event. Our study shows that the behaviour of the relativistic electron population can take three distinctly different forms, which are strongly correlated with the solar wind conditions during the storm recovery phase.

\section{Instrumentation}

Our measurements of the relativistic electron population were made by instruments aboard the two microsatellites, Space Technology Research Vehicle-1a (STRV-1a) and STRV-1b. These UK spacecraft were launched together into geostationary transfer orbit on 17 June 1994 with a perigee of $300 \mathrm{~km}$ altitude, an apogee of $36000 \mathrm{~km}$ altitude, an inclination of $7^{\circ}$ and a period of $10.58 \mathrm{~h}$. STRV-1a and STRV$1 \mathrm{~b}$ continued to operate up until March 1998 and September 1998, respectively, at which points in time the instruments were turned off. The satellites traversed the magnetospheric equatorial region about four times per day, moving between $L=1.1$ out to $L \approx 7$. The two satellites were in very similar orbits, but had slightly different speeds and thus 
varying separation. Electron count rates are obtained from the Cold Ion Detector (CID) (Papatheodorou et al., 1996) on board STRV-1a and from the Radiation Environment Monitor (REM) (Bühler et al., 1996) on board STRV-1b. The CID instrument is a microchannel plate-based detector shielded by $2 \mathrm{~mm}$ of aluminium giving it a minimum energy threshold of approximately $750 \mathrm{keV}$, whereas the REM instrument is a silicon diode detector and has a $3 \mathrm{~mm}$ aluminium shield resulting in a higher minimum energy threshold near $1 \mathrm{MeV}$. The instruments each provide a measure of the number of electrons above their respective energy thresholds. The CID has a lower energy threshold, but typically records a lower count rate than the REM, due to the difference in geometric factors of the two instruments. The adiabatic invariants are the natural choice of parameters with which to describe the relativistic electron population distribution. Unfortunately, due to their fixed energy thresholds, the detectors will see varying proportions of the adiabatic parameter space as the spacecraft travel around their orbits, due to the variation of the local magnetic field strength around the orbit. Nevertheless, to a first approximation this proportion will be a timeindependent function of $L$. Thus, by integrating over a range of $L(3.5<L<6.5)$, we can compare changes in the entire relativistic electron population over time that will properly indicate significant enhancements or losses. The instruments detect particles of all pitch angles, but do not resolve them by pitch angle.

\section{Observations and results}

A relative measure of the Total Relativistic Electron Content $\left(T_{\mathrm{REC}}\right)$ in the outer radiation belt is calculated separately for both the CID and the REM in the range $L=3.5$ to $L=6.5$ for each traverse of the radiation belts made by the satellites. Assuming dipole field geometry and using spherical polar coordinates, the free space volume enclosed by a given L-shell is given by

$\nu=2 \int_{0}^{2 \pi} \int_{\theta_{\min }}^{\theta_{\max }} \int_{R_{\min }}^{R_{\max }} r^{2} \sin \theta d r d \theta d \phi$,

where $R_{\min }=R_{0}, R_{\max }=L R_{0} \sin ^{2} \theta$ and $\theta_{\min }=\pi / 2$ $\cos ^{-1}(1 / L)^{1 / 2}$ and $\theta_{\max }=\pi / 2$. In the region $3.5<L<6.5$ this reduces to a very good approximation to $V \approx 1.914$ $L^{3} R_{0}^{3}$, (maximum error at $L=3.5$ of $<5 \%$ ). The volume occupied between dipolar shells at $L$ and $L+\Delta L$ may then be written as:

$V_{\text {shell }} \approx 5.743 R_{0}^{3} L^{2} \Delta L$.

To calculate a relative measure of the relativistic electron content within the outer radiation belt, the count rate is initially binned for the CID and for the REM, as a function of $L$ by averaging in steps of $\Delta L=0.2$, giving $C(L)$ for each instrument. A relative measure of the electron content within a notional drift shell of radius $L$ in the equatorial plane and thickness $\Delta L=0.2, T(L)$, is then approximated by $T(L)=C(L) \times L^{2}$, where the factor $L^{2}$ makes an allowance for the volume occupied between dipolar shells at $L$ and $L+\Delta L$, as explained above. A relative measure of the Total Relativistic Electron Content $\left(T_{\mathrm{REC}}\right)$ in the outer radiation belt is then obtained for each instrument by summing $T(L)$ between $3.5<L<6.5$. We have assumed that the magnetic field geometry can be approximated to a symmetrical dipole, that the relativistic electron distribution is longitudinally symmetric, that mirror point separation distances measured along the field line scale as $L$, and the pitch angle distribution is isotropic. The true situation may be somewhat different, particularly the magnetic field geometry, but the weighting by $L^{2}$ is a reasonable first approximation.

Figure 1 shows a comparative plot of $T_{\mathrm{REC}}$ and solar wind and geomagnetic parameters. The period shown is from 1 January 1995 until 30 June 1995 during the declining phase of solar cycle 22, a period characterised by recurrent high speed solar wind streams. Figure 1 shows both $T_{\mathrm{REC}}$ and the position in $L$, at which the CID and the REM detected the maximum $T(L)$ during each pass through the outer radiation belt. These results are compared with the solar wind velocity, the interplanetary magnetic field (IMF) $z$-component and the solar wind dynamic pressure obtained from the WIND spacecraft (Ogilvie et al., 1995; Farrel et al., 1995). The solar wind speed was smoothed using a Gaussian shaped boxcar average with a six hour window as a suitable averaging technique to reduce noise. We present $D_{s t} *$ (pressure corrected $D_{s t}$ ) at one-hourly values as a measure of the magnetospheric response. Using $D_{s t} *$ removes variations in $D_{s t}$ caused by magnetopause currents induced by solar wind pressure perturbations, thus providing a more accurate measure of the ring current. The correction is given by

$D_{s t}{ }^{*}=D_{s t}-7.26 \sqrt{P_{\mathrm{dyn}}}+11$,

where $P_{\mathrm{dyn}}$ is the solar wind dynamic pressure (O'Brien and Mc Pherron, 2000).

Figure 1 shows the variation of the relativistic electron population during the 17 magnetic storms. The criteria for the selection of a magnetic storm event was a minimum $D_{s t}$ * $<-40 \mathrm{nT}$. In addition, we required that the $D_{s t} *$ index had returned to $\sim 0 \mathrm{nT}$ prior to the next storm onset, ensuring separation between events. Sometimes, but not always, the magnetic storm recovery phase is accompanied by a sustained increase in $T_{\mathrm{REC}}$. In order to properly compare the pre-storm $T_{\mathrm{REC}}$ with the post-storm $T_{\mathrm{REC}}$, it is necessary that the comparison is made when the geomagnetic field conditions in the outer radiation belt are the same (i.e. when $D_{s t} *$ is approximately the same), so as to rule out changes in the measured electron count rate due to the electrons' adiabatic response to changing geomagnetic field conditions during the storm.

Over the six-month period, three different types of responses of the relativistic electron population during a storm were identified. In general, all three classes of events begin with an extended period of (often strong) southward IMF that leads to the magnetic storm main phase and a rapid decrease in $T_{\mathrm{REC}}$ above $750 \mathrm{keV}$. The differences between the 

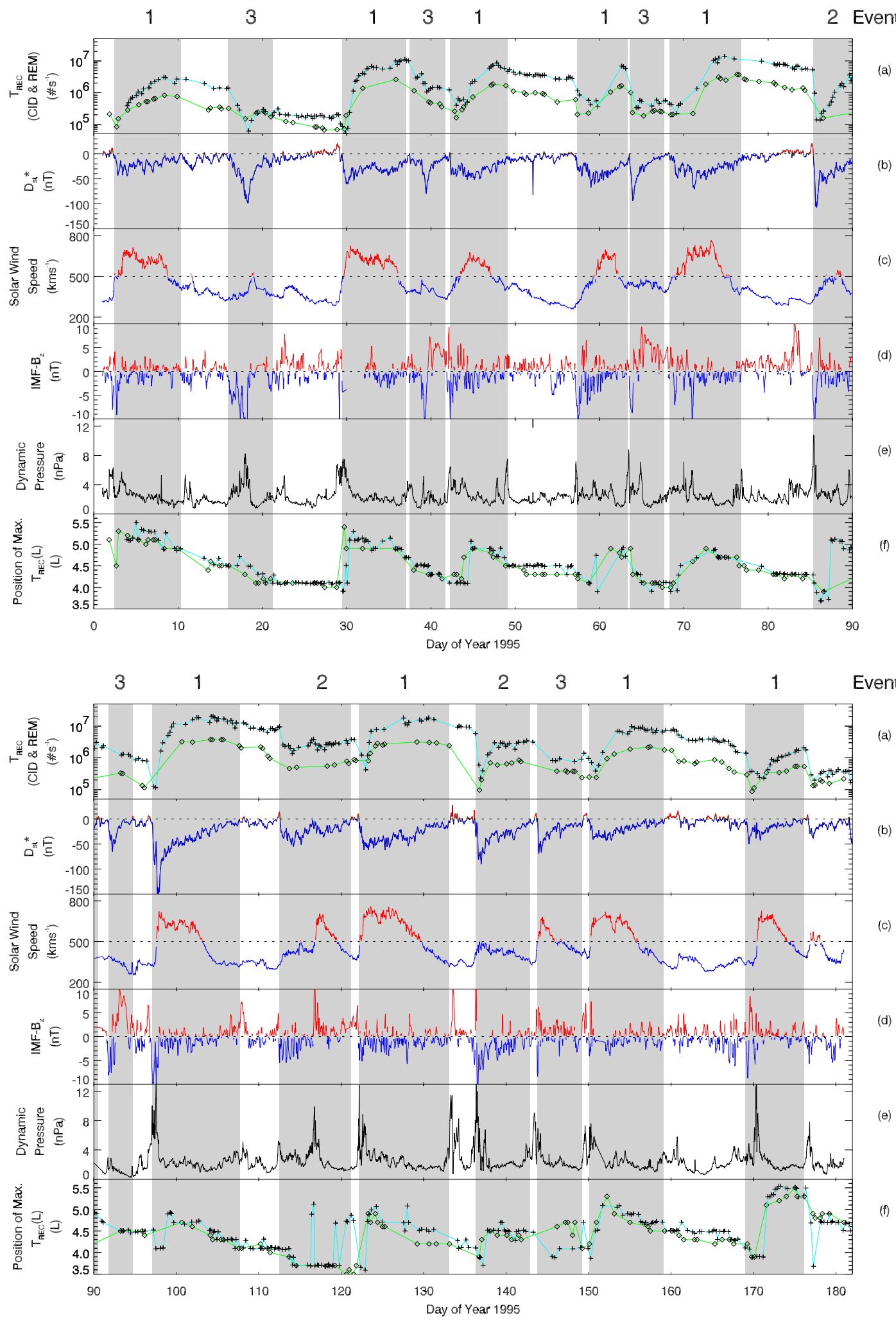

Fig. 1. The relativistic electron response during magnetic storms is compared with solar wind and geomagnetic parameters during the first six months of 1995. The panels show (a) a relative measure of the Total Relativistic Electron Content ( $T_{\mathrm{REC}}$ ) in the range $3.5<L<6.5$, REM (blue line), CID (green line); (b) $D_{s t} *(\mathrm{nT})$; (c) the solar wind speed $\left(\mathrm{km}^{-1}\right)$; (d) IMF- $B_{z}(\mathrm{nT})$; (e) the solar wind dynamic pressure $(\mathrm{nPa})$; (f) and the position of the maximum $T_{\mathrm{REC}}(L)$ in the outer radiation belt $(L)$. The shaded areas define the magnetic storms studied with the event type indicated along the top. 
Table 1. The day of year and value of the $D_{s t} *$ minimum for each event, together with the pre- and post-storm $T_{\mathrm{REC}}$ for the CID measured at the beginning and end of each storm interval and the associated event type and change in $T_{\mathrm{REC}}$

\begin{tabular}{c|c|c|c|c|c|c|c}
\hline $\begin{array}{c}\text { Magnetic } \\
\text { storm }\end{array}$ & $\begin{array}{c}D_{s t} * \\
\text { minimum }\end{array}$ & \multicolumn{2}{|c|}{ Pre-Storm } & \multicolumn{2}{c}{ Post-Storm } & Type & $\Delta T_{\text {REC }}$ \\
$\begin{array}{c}\text { Day of } \\
\text { Year }\end{array}$ & $(\mathrm{nT})$ & $(\mathrm{DoY})$ & $\begin{array}{c}T_{\mathrm{REC}} \\
(\# / \mathrm{s})\end{array}$ & $\begin{array}{c}\text { Time } \\
(\mathrm{DoY})\end{array}$ & $\begin{array}{c}T_{\mathrm{REC}} \\
(\# / \mathrm{s})\end{array}$ & & $(\# / \mathrm{s})$ \\
\hline 2.8 & -42.3 & 1.8 & $2.1 \times 10^{5}$ & 9.9 & $7.5 \times 10^{5}$ & 1 & $5.45 \times 10^{5}$ \\
18.3 & -96.5 & 15.9 & $3.1 \times 10^{5}$ & 21.0 & $1.7 \times 10^{5}$ & 3 & $-1.45 \times 10^{5}$ \\
30.0 & -57.7 & 27.3 & $6.5 \times 10^{4}$ & 37.2 & $1.5 \times 10^{6}$ & 1 & $1.39 \times 10^{6}$ \\
39.4 & -80.7 & 37.2 & $1.5 \times 10^{6}$ & 41.7 & $3.1 \times 10^{5}$ & 3 & $-1.14 \times 10^{6}$ \\
45.1 & -54.2 & 41.7 & $3.1 \times 10^{5}$ & 48.9 & $1.7 \times 10^{6}$ & 1 & $1.34 \times 10^{6}$ \\
58.9 & -67.7 & 57.1 & $6.0 \times 10^{5}$ & 63.3 & $1.2 \times 10^{6}$ & 1 & $5.50 \times 10^{5}$ \\
63.9 & -92.3 & 63.3 & $1.2 \times 10^{6}$ & 68.4 & $1.9 \times 10^{5}$ & 3 & $-9.60 \times 10^{5}$ \\
71.2 & -72.8 & 68.4 & $2.0 \times 10^{5}$ & 76.7 & $3.4 \times 10^{6}$ & 1 & $3.21 \times 10^{6}$ \\
85.7 & -108.4 & 84.4 & $1.2 \times 10^{6}$ & - & - & 2 & - \\
92.3 & -68.6 & - & - & 93.5 & $3.1 \times 10^{5}$ & 3 & - \\
97.7 & -146.6 & 95.8 & $1.3 \times 10^{5}$ & 107.6 & $2.5 \times 10^{6}$ & 1 & $2.33 \times 10^{6}$ \\
114.2 & -55.0 & 111.4 & $9.0 \times 10^{5}$ & 121.6 & $8.5 \times 10^{5}$ & 2 & $-5.00 \times 10^{4}$ \\
123.3 & -59.2 & 121.6 & $8.5 \times 10^{5}$ & 133.0 & $2.4 \times 10^{6}$ & 1 & $1.50 \times 10^{6}$ \\
137.0 & -94.0 & 133.0 & $2.4 \times 10^{6}$ & 141.8 & $7.5 \times 10^{5}$ & 2 & $-1.60 \times 10^{6}$ \\
144.0 & -66.0 & 141.8 & $7.5 \times 10^{5}$ & 149.1 & $2.4 \times 10^{5}$ & 3 & $-5.15 \times 10^{5}$ \\
152.1 & -47.4 & 149.1 & $2.4 \times 10^{5}$ & 157.2 & $2.2 \times 10^{6}$ & 1 & $1.92 \times 10^{6}$ \\
169.4 & -44.8 & 169.2 & $2.9 \times 10^{5}$ & 175.0 & $5.5 \times 10^{5}$ & 1 & $2.60 \times 10^{5}$ \\
\hline
\end{tabular}

events become apparent when we consider what follows during the recovery phase of the magnetic storm. We classify a "Type 1 " event as having a post-storm $T_{\text {REC }}$ greater than the pre-storm $T_{\text {REC }}$ (comparing count rates measured at times of equal $D_{s t} *$, before and after the $D_{s t} *$ minimum). Similarly, a "Type 2 " event is identified by a partial recovery of $T_{\mathrm{REC}}$ following the storm main phase, which does not exceed the pre-storm $T_{\text {REC }}$ value. The "Type 3" event is characterised by a failure of $T_{\text {REC }}$ to recover following the storm main phase. In Fig. 1, the storm intervals are shaded grey and annotated according to event type. The pre- and post-storm $T_{\text {REC }}$ values for the CID and the REM for each event are measured at the beginning and end of each storm interval and are tabulated in Table 1 and 2, respectively, together with the $D_{s t}{ }^{*}$ minimum, event type and change in $T_{\mathrm{REC}}$.

In the nine Type 1 events an extended interval (two days or more) of persistently high solar wind speed, typically at levels of greater than $500 \mathrm{~km} \mathrm{~s}^{-1}$, is seen during the storm recovery phase (defined by $D_{s t}{ }^{*}$ ). Moreover, the IMF $B_{z}$ is either predominantly southward or fluctuating about zero. During the period of fast solar wind, the $T_{\mathrm{REC}}$ enhancement occurs as a steady build up which apparently goes on as long as the solar wind speed remains high and the IMF $B_{z}$ is southward or at least fluctuating.

In the four Type 2 events, the initial conditions are similar to those in Type 1. There is an increase in solar wind speed following the storm main phase, as before, however it is generally smaller and shorter-lived than for Type 1 events, in most cases not exceeding $500 \mathrm{~km} \mathrm{~s}^{-1}$. Once again, the IMF $B_{z}$ is either southward or fluctuating about zero during the recovery phase. The $T_{\mathrm{REC}}$ begins to increase during the interval of raised solar wind speed during the recovery phase, but in these cases, the final $T_{\text {REC }}$ does not reach the pre-storm $T_{\text {REC }}$ level. The outcome appears to be a lesser degree of electron flux enhancement than in Type 1 events as a result of a slower solar wind speed.

Evidence of a localised region of the greatest $T_{\mathrm{REC}}$ enhancement is seen in both Type 1 and Type 2 events as a new peak of the electron distribution forms in the region of $L=4.5$ to $L=5.5$. The location of the new peak in $T(L)$ is at smaller $L$ for storms with more negative $D_{s t} *$ minima see Fig. 2. Following the $T_{\text {REC }}$ enhancement the position of the peak in $T(L)$ gradually drifts inward.

In the five Type 3 events, the IMF conditions leading up to the magnetic storm are the same as in the previous two cases, most notably an extended period of (often) strong southward IMF $B_{z}$ that leads to the magnetic storm main phase. However, during the magnetic storm recovery phase, the electron count rate remains low and the peak of the distribution either stays where it is or continues to drift inwards, in contrast to the appearance of enhanced $T_{\text {REC }}$ values seen between $L=4.5$ to $L=5.5$ in Type 1 and 2 events. Although 


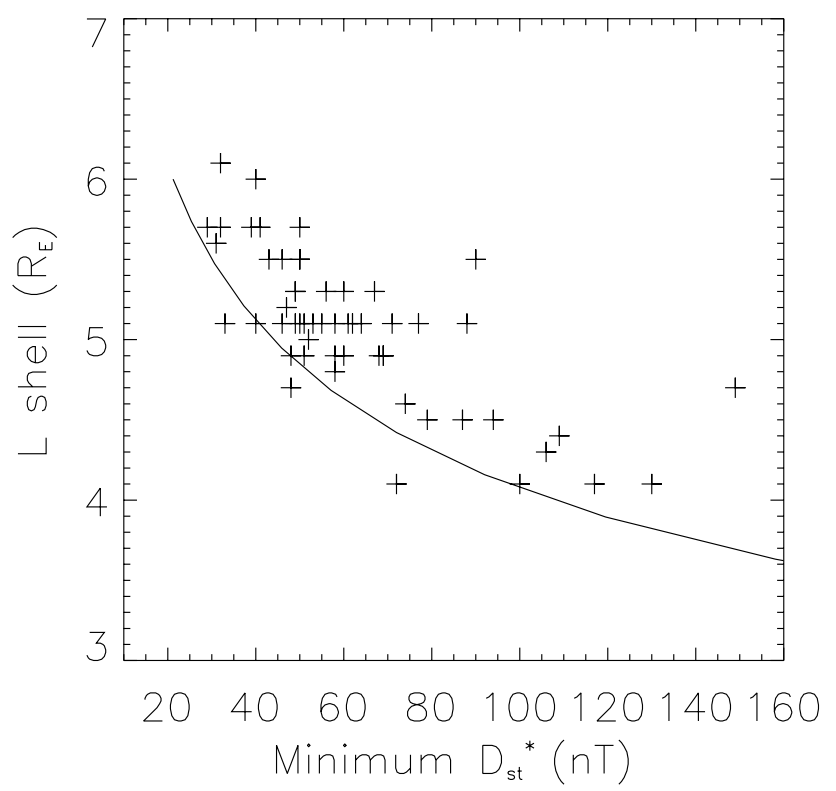

Fig. 2. Correlation between the position of the maximum $T(L)$ in the outer radiation belt during the period of largest flux enhancement with the minimum $D_{s t} *$ value of the coincident magnetic storm. Results from CID data between January 1995 to March 1998 . Correlation coefficient $=-0.728584$. The curved line is the relationship derived by Tverskaya (1996). $\left|D_{s t}\right|_{\max }=2.75 \times 10^{4} / L_{\max }^{4}$.

the solar wind speed in some cases approaches or exceeds $500 \mathrm{~km} \mathrm{~s}^{-1}$, no enhancement in $T_{\mathrm{REC}}$ takes place. This difference in the electron response compared to the previous two cases appears to be due to a difference in the IMF $B_{z}$ orientation during the magnetic storm recovery phase. Following the initial period of southward IMF $B_{z}$ that is the cause of the magnetic storm, $B_{z}$ turns northward and remains predominantly and often strongly northward during the period that the electron flux enhancement might otherwise be taking place. It should also be noted that $D_{s t} *$ generally has a much more rapid recovery phase $\left(D_{s t} *\right.$ returns to $\left.0 \mathrm{nT}\right)$ in the Type 3 events than in the Type 1 and 2 events.

Figure 2 shows the relationship between the position of the maximum $T_{\mathrm{REC}}$ during the period of largest flux enhancement and the minimum $D_{s t} *$ of the coincident magnetic storm. The results are for 55 different events using the CID data from January 1995 up until March 1998. The correlation shows that for stronger magnetic storms, the position of the maximum enhanced $T_{\mathrm{REC}}$ is found to occur at lower L-shells. Also shown in Fig. 2 is the empirical solution found by Tverskaya (1996), based on many measurements of $D_{s t}$ and the L-shell during the formation of the new peak in the outer radiation belt and is given by $\left|D_{s t}\right|_{\max }=2.75$ $\times 10^{4} / L_{\max }^{4}$. The two results show a fairly good correspondence, however, the solution found by Tverskaya tends to lie slightly below the results from the CID. This difference is probably a result of the difference between the approximation of the relativistic electron content $(T(L))$ used in Fig. 2 and the relation found by Tverskaya, which is based on local count rate measurements. The $L^{2}$ weighting used in the calculation of the electron content on a given drift shell will bias the $T(L)$ to have higher values at larger L-shells. As a consequence, the peak of the radiation belt is systematically shifted to a larger L-value. By acknowledging this difference we feel it is interesting to note that both results display a similar trend thus further supporting a $D_{s t} *$ versus $L$ relationship. However, the measure of the position of the peak of the radiation belt measured by $T(L)$ should give a more realistic indication of where the maximum amount of electron acceleration has occurred.

\section{Discussion}

The typical magnetospheric response during a magnetic storm can be described as follows. The intensification of the ring current during the magnetic storm main phase leads to a drop in the magnetic field strength earthward of the ring current. The relativistic electrons respond adiabatically to the decrease in the magnetic field strength and move outward while at the same time losing energy, in what is known as the $D_{s t}$ effect. The increased magnetospheric wave activity and subsequent wave-particle interactions lead to an increase in the rate of electron loss as they are scattered into the loss cone (Lyons et al., 1972). Electrons on the outermost drift paths also become more susceptible to being lost as many end up on drift paths that intersect the dayside magnetopause (Desorgher et al., 2000). During the recovery phase the electrons that have not been permanently lost will return to their original state, if no other acceleration or transportation has occurred (Kim and Chan, 1997).

Observations of the relativistic electron population shown here are consistent with the idea that significant loss of energetic electrons occurs in every storm (Desorgher et al., 2000; Kim and Chan, 1997), in some cases removing the majority of the relativistic electron population. This electron loss appears to be more efficient at higher energies, as seen by the generally larger decrease of the $>1 \mathrm{MeV} T_{\mathrm{REC}}$ observed by the REM, in comparison with the $>750 \mathrm{keV} T_{\mathrm{REC}}$ detected by the CID. Brief periods of decreased count rates associated with the onset of magnetic storms may be due simply to an adiabatic process in response to the pressure pulse (Blake et al., 1997). However, the results in Fig. 1 show that solitary pressure pulses do not produce themselves significantly reduced count rates. A clear example can be seen when the effect on $T_{\text {REC }}$ during the pressure pulse on Day 133 is compared with the following pressure pulse on Day 136. It appears that the major decreases in the electron count rate are generally associated with a magnetic storm main phase. It is possible to confirm that real losses of energetic electrons occur during the storms under examination when the $T_{\mathrm{REC}}$ levels are compared at times of similar $D_{s t}{ }^{*}$, in order to remove the influence of an adiabatic response to the altered magnetic field configuration during the storm. This is most clearly seen when comparing the pre- and post- $T_{\mathrm{REC}}$ levels 
Table 2. The day of year and value of the $D_{s t} *$ minimum for each event, together with the pre- and post-storm $T_{\mathrm{REC}}$ for the REM measured at the beginning and end of each storm interval and the associated event type and change in $T_{\mathrm{REC}}$

\begin{tabular}{|c|c|c|c|c|c|c|c|}
\hline \multirow{2}{*}{$\begin{array}{c}\text { Magnetic } \\
\text { storm } \\
\text { Day of } \\
\text { Year }\end{array}$} & \multirow{2}{*}{$\begin{array}{c}D_{s t} * \\
\text { minimum }\end{array}$} & \multicolumn{2}{|c|}{ Pre-Storm } & \multicolumn{2}{|c|}{ Post-Storm } & \multirow[t]{2}{*}{ Type } & \multirow{2}{*}{$\begin{array}{c}\Delta T_{\mathrm{REC}} \\
(\# / \mathrm{s})\end{array}$} \\
\hline & & $\begin{array}{l}\text { Time } \\
\text { (DoY) }\end{array}$ & $\begin{array}{c}T_{\mathrm{REC}} \\
(\# / \mathrm{s}) \\
(\mathrm{REM})\end{array}$ & $\begin{array}{l}\text { Time } \\
\text { (DoY) }\end{array}$ & $\begin{array}{c}T_{\text {REC }} \\
(\# / \mathrm{s}) \\
(\mathrm{REM})\end{array}$ & & \\
\hline 2.8 & -42.3 & - & - & 9.9 & $2.8 \times 10^{6}$ & 1 & - \\
\hline 18.3 & -96.5 & 15.9 & $1.3 \times 10^{6}$ & 21.2 & $1.9 \times 10^{5}$ & 3 & $-1.11 \times 10^{6}$ \\
\hline 30.0 & -57.7 & 28.2 & $1.6 \times 10^{5}$ & 36.9 & $1.1 \times 10^{7}$ & 1 & $1.09 \times 10^{7}$ \\
\hline 39.4 & -80.7 & 37.2 & $1.0 \times 10^{7}$ & 41.4 & $1.3 \times 10^{6}$ & 3 & $-8.70 \times 10^{6}$ \\
\hline 45.1 & -54.2 & 41.1 & $1.4 \times 10^{6}$ & 49.2 & $5.5 \times 10^{6}$ & 1 & $4.10 \times 10^{6}$ \\
\hline 58.9 & -67.7 & 56.2 & $3.1 \times 10^{6}$ & 62.8 & $6.5 \times 10^{6}$ & 1 & $3.40 \times 10^{6}$ \\
\hline 63.9 & -92.3 & 62.8 & $6.5 \times 10^{6}$ & 67.8 & $5.5 \times 10^{5}$ & 3 & $-5.95 \times 10^{6}$ \\
\hline 71.2 & -72.8 & 67.8 & $5.5 \times 10^{5}$ & 76.1 & $1.2 \times 10^{7}$ & 1 & $2.00 \times 10^{7}$ \\
\hline 85.7 & -108.4 & 84.7 & $5.5 \times 10^{6}$ & 91.0 & $2.0 \times 10^{6}$ & 2 & $-3.50 \times 10^{6}$ \\
\hline 92.3 & -68.6 & 91.1 & $2.5 \times 10^{6}$ & 94.5 & $1.1 \times 10^{6}$ & 3 & $-1.40 \times 10^{6}$ \\
\hline 97.7 & -146.6 & 96.4 & $8.0 \times 10^{5}$ & 106.6 & $1.5 \times 10^{7}$ & 1 & $1.42 \times 10^{7}$ \\
\hline 114.2 & -55.0 & 112.2 & $8.5 \times 10^{6}$ & 121.2 & $3.8 \times 10^{6}$ & 2 & $-4.75 \times 10^{6}$ \\
\hline 123.3 & -59.2 & 121.2 & $3.8 \times 10^{6}$ & 134.6 & $9.0 \times 10^{6}$ & 1 & $5.25 \times 10^{6}$ \\
\hline 137.0 & -94.0 & 134.6 & $9.0 \times 10^{6}$ & 142.6 & $3.1 \times 10^{6}$ & 2 & $-5.90 \times 10^{6}$ \\
\hline 144.0 & -66.0 & 142.6 & $3.1 \times 10^{6}$ & 149.1 & $9.0 \times 10^{5}$ & 3 & $-2.20 \times 10^{6}$ \\
\hline 152.1 & -47.4 & 149.1 & $9.0 \times 10^{5}$ & 157.6 & $8.0 \times 10^{6}$ & 1 & $7.10 \times 10^{6}$ \\
\hline 169.4 & -44.8 & 169.1 & $1.4 \times 10^{6}$ & 176.1 & $2.0 \times 10^{6}$ & 1 & $6.00 \times 10^{5}$ \\
\hline
\end{tabular}

in the Type 3 events. Similar conditions may have existed for many other reported magnetic storms that did not lead to an enhancement in the electron count rate, such as those reported by Reeves (1998). It seems reasonable to assume that real losses have in fact occurred in all cases, Types 1 to 3 . The count rate enhancements in Type 1 and Type 2 events thus appear to result from new energetic particles appearing in the magnetosphere in sufficient numbers to replace most or all of the particles lost earlier during the storm.

The important difference between the event types occurs during the recovery phase of the magnetic storms, not during the onset or main phase. The magnitude and duration of negative $B_{z}$ prior to the recovery phase may effect the strength of the subsequent magnetic storm and also the proportion of particles lost, but it does not significantly effect the size of the enhancement in $T_{\mathrm{REC}}$ during the recovery phase. Both Type 1 and Type 2 events typically have a fluctuating or negative $B_{z}$ during the recovery phase, thus the difference between these two event types lies in the solar wind speed. The Type 1 events are associated with a more prolonged and often larger increase in the solar wind speed than the Type 2 events, and as a result, have larger enhancements in the electron count rate. The important difference between the Type 3 events and the Type 1 and 2 events is that the IMF $B_{z}$ is predominantly northward during the recovery phase of Type 3 events in contrast to a southward or fluctuating about zero
$B_{z}$ during the other two event types. It is this difference in the orientation of the IMF $B_{z}$ during the magnetic storm recovery phase that is most important and appears to determine whether the magnetic storm will result in an enhancement in the relativistic electron count rate (southward/fluctuating $B_{z}$ ) or not (northward $B_{z}$ ). The orientation of the IMF $B_{z}$ appears to act as a switch, either turning-off the enhancement when northward during the recovery phase or allowing the enhancement in the count rate to proceed for southward or fluctuating $B_{z}$.

Blake et al. (1997) studied the relativistic electron response in two energy channels ( $E>1.5 \mathrm{MeV} ; E>3.5 \mathrm{MeV})$ during the same time period using detectors on board the spacecraft 1994-026. Although the spacecraft 1994-026 and the two STRV satellites have similar apogees and perigees, the STRV spacecraft orbital plane has a $7^{\circ}$ inclination, which is much closer to the magnetic equator than the $62^{\circ}$ inclination of 1994-026. The STRV spacecraft, therefore, see electrons of virtually all equatorial pitch angles for most of the time across the full L-shell range of their orbit, whereas the equatorial pitch angle coverage of 1994-026 will change significantly around the orbit and, in particular, will be less complete at larger $L$. Across $L$, the general trend of the relativistic electron count rates seen by STRV-1a, STRV- $1 \mathrm{~b}$ and 1994-026, are similar at all times, showing that the process of electron flux enhancement has not been confined to a narrow 
pitch angle range.

Blake et al. (1997) select five case studies associated with the large, abrupt increases in the solar wind speed on days, 97, 113, 117, 123, and 136 in 1995. They emphasize the significance of a southward turning IMF, which is seen as a switch enabling "a high speed stream and leading pressure pulse to have a strong effect on the energetic population". Conversely, they state that the effect of a high speed stream is nil when associated with a clearly northward turning field. In contrast, the selection of the events used in our study is based on the identification of magnetic storms according to $D_{s t}{ }^{*}$. In almost all cases, a significant pressure pulse and an interval of southward IMF precede the magnetic storm. Thus, the conclusion drawn is similar to that of Blake et al. (1997) but not identical; a southward IMF can be identified as a precursor to all the storms in this study, including those with no enhancement in $T_{\mathrm{REC}}$, and so it appears to be the behaviour of the IMF $B_{z}$ in the interval following the magnetic storm main phase that controls whether a high speed stream can affect the magnetospheric electrons. It is also necessary that a high speed stream be observed during the recovery phase, in order to see a significant and prolonged energetic electron enhancement; a pressure pulse alone is insufficient.

Extended periods of southward IMF $B_{z}$ in the recovery phase will be associated with prolonged substorm activity. The substorm injected electrons may play a role in the acceleration process, by producing a seed population of electrons with energies of a few hundred $\mathrm{keV}$ which may then be subsequently accelerated to $\mathrm{MeV}$ energies (e.g. Obara et al., 2000). Furthermore, the injection of anisotropic medium energy (10-100 keV) electrons during substorms (Baker et al., 1998) leads to the excitation of intense whistler mode waves which have been postulated as a possible generator of $\mathrm{MeV}$ electrons (Horne and Thorne, 1998; Summers et al., 1998; Summers and Ma, 2000). Whistler mode waves are known to be substorm-dependent (e.g. Tsuratani and Smith, 1977; Meredith et al., 2001) and have recently been observed in association with the generation of outer zone relativistic electrons (Meredith et al., 2002a, 2002b), lending credence to this proposed mechanism. However, ULF waves have also been invoked as a means of accelerating the seed electrons to $\mathrm{MeV}$ energies. Enhanced ULF waves are correlated with high solar wind speeds (Mathie and Mann, 2001) and have been associated with the generation of relativistic electrons at geosynchronous orbit (Mathie and Mann, 2000). Our work has shown that significant enhancements in $T_{\mathrm{REC}}$ are correlated with periods of prolonged substorm activity and high speed solar wind during the recovery phase. These conditions are likely to lead to the generation of intense whistler mode waves and ULF waves, making it difficult to discriminate between the two mechanisms on this observation alone.

A prolonged increase in the solar wind speed beyond $\sim 500 \mathrm{~km} \mathrm{~s}^{-1}$ may drive a number of potential electron acceleration mechanisms within the outer radiation belt. The ULF waves invoked in the drift-resonance (Elkington et al., 1999) or global magnetic pumping (Liu et al., 1999) mechanisms may be generated via Kelvin-Helmoltz instabilities along the magnetopause created by fast magnetosheath flow speeds (perhaps $>500 \mathrm{~km} \mathrm{~s}^{-1}$; Mann et al., 1999; Mann and Wright, 1999). In addition, the whistler mode chorus waves invoked in the potential gyro-resonant electron acceleration (Meredith et al., 2002a; Summers and Ma, 2000) have a strong dependence on substorm activity (Meredith et al., 2001). A prolonged increase in the solar wind speed during the recovery phase of a magnetic storm during which $B_{z}$ is negative or fluctuating about zero will lead to increased substorm activity and hence stronger plasma wave intensities.

The formation of a new peak in the outer radiation belt during the recovery phase of Type 1 and Type 2 events is evidence for local electron acceleration. The new peak typically forms immediately after the storm main phase, during which there is a significant injection of lower energy electrons (tens to a few hundred $\mathrm{keV}$ ) into the outer radiation belt (Baker et al., 1998). If these electrons are energised to $\mathrm{MeV}$ energies in a local acceleration process, then the maximum enhancement in the count rate and hence the new peak will appear at the same $L$ to which this lower energy population is injected during the storm main phase. The $D_{s t} *$ versus $L$ relationship then arises as a natural consequence of the lower energy electrons being injected deeper into the outer zone during a more enhanced convection electric field during stronger magnetic storms.

The $D_{s t} *$ versus $L$ relationship lends further support to the local stochastic electron acceleration by whistler mode waves. In contrast, the process of electron acceleration via the drift-resonance mechanism, that invokes ULF waves, provides energetic electrons to the inner magnetosphere from a source beyond the outer radiation belt. It is, therefore, difficult to reconcile the proposed drift-resonance electron acceleration with the observed relationship between the strength of a magnetic storm and the radial location of the subsequent $T_{\text {REC }}$ enhancement.

The subsequent inward movement of the peak $T_{\text {REC }}$ during the recovery phase may be caused by inward radial diffusion, combined with increased rates of electron loss at larger L-values. However, continuing in situ electron acceleration in this region of the outer radiation belt may also cause the inward movement of the peak $T_{\mathrm{REC}}$.

\section{Conclusions}

We have used data from the STRV microsatellites during the first six months of 1995 to study the magnetospheric relativistic electron response during magnetic storms. The main results of this study are that:

(a) an extended interval (two days or more) of fast solar wind speed above $\sim 500 \mathrm{~km} \mathrm{~s}^{-1}$ and an IMF- $B_{z}$ that is fluctuating about zero or more predominantly southward during the magnetic storm recovery phase are the main requirements for significant electron enhancements at relativistic energies; 
(b) predominantly northward IMF during the recovery phase severely limits the recovery of the relativistic electrons;

(c) the location of the peak in $T_{\mathrm{REC}}$ during the storm recovery phase is dependent on the strength of the magnetic storm;

(d) particle loss during the magnetic storm main phase often dominates the $D_{s t}$ effect, in some cases leading to no recovery of the electron $T_{\mathrm{REC}}$ through the $D_{s t}$ effect following the storm main phase.

Acknowledgements. We thank the World Data Centre for Geomagnetism C2 for providing the $D_{s t}$ index and CDAWeb for the WIND key-parameters used in this paper. R.H.A.I is supported by a PPARC studentship.

Topical Editor G. Chanteur thanks T. Nagatsuma and another referee for their help in avaluating this paper.

\section{References}

Baker, D. N., Li, X., Blake, J. B., and Kanekal, S.: Strong Electron Acceleration in the Earth's Magnetosphere, Adv. Space Res., 21, 4, 609-613, 1998.

Blake, J. B., Baker, D. N., Turner, N., Ogilvie, K. W., and Lepping, R. P.: Correlation of changes in the outer-zone relativisticelectron population with upstream solar wind and magnetic field measurements, Geophys. Res. Lett., 24, 927, 1997.

Bühler, P., Ljungfelt, S., Mchedlishvili, A., Schlumpf, N., Zehnderm, A., Adamsm, L., Daly, E., and Nickson, R.: Radiation environment monitor, Nucl. Instrum, Methods Phys. Res., Sect. A, 368, 825, 1996

Desorgher, L., Bühler, P., Zehnder, A., and Flückiger, E. O.: Simulations of the outer radiation belt electron flux decrease during the March 26, 1995, magnetic storm, J. Geophys. Res., 105, A9, $21211,2000$.

Elkington, S. R., Hudson, M. K., and Chan, A. A.: Acceleration of relativistic electrons via drift-resonant interaction with toroidalmode Pc5 ULF oscillations, Geophys. Res. Lett., 26(21), 3273$3276,1999$.

Farrell, W. M., Thompson, R. F., Lepping, R. P., and Byrnes, J. B.: A method of calibrating magnetometers on a spinning spacecraft, IEEE Transactions on Magnetics, 31(2), 966-972, 1995.

Horne, R. B. and Thorne, R. M.: Potential waves for relativistic electron scattering and stochastic acceleration during magnetic storms, Geophys. Res. Lett., 25, 3011, 1998.

Horne, R. B., Meredith, N. P., Thorne, R. M., Heyndeerickx, D., Iles, R. H. A., and Anderson, R. R.: Evolution of energetic electron pitch angle distributions during storm time electron acceleration to MeV energies, J. Geophys. Res., submitted, 2001.

Kim, H.-J. and Chan, A. A.: Fully adiabatic changes in storm time relativistic electron fluxes, J. Geophys. Res., 102, 22 107, 1997.

Li, X. and Temerin, M. A.: The electron radiation belt, Space Sci. Rev., 95, 569, 2001.

Liu, W. W., Rostoker, G., and Baker, D. N.: Internal acceleration of relativistic electrons by large amplitude ULF pulsations, J. Geo- phys. Res., 104 (A8), 17 391-17 407, 1999.

Lyons, L. R., Thorne, R. M., and Kennel, C. F.: Pitch-angle diffusion of radiation belt electrons within the plasmasphere, J. Geophys. Res., 77, 3455, 1972.

Ogilvie, K. W., Chornay, D. J., Fritzenreiter, R. J., Hunsaker, F., Keller, J., Lobell, J., Miller, G., Scudder, J. D., Sittler, E. C., Torbert, R. B., Bodet, D., Needell, G., Lazarus, A. J., Steinberg, J. T., and Tappan, J. H.: SWE, A comprehensive plasma instrument for the WIND spacecraft, Space Sci. Rev., 71,(1-4), 55-77, 1995.

Mann, I. R. and Wright, A. N.: Diagnosing the excitation mechanisms of Pc5 magnetospheric flank waveguide modes and FLRs, Geophys. Res. Lett, 16 (16), 2609-2612, 1999.

Mann, I. R., Wright, A. N., Mills, K. J., and Nakariakov, V. M.: Excitation of magnetospheric waveguide modes by magnetosheath flows, J. Geophys. Res., 104 (A1), 333-353, 1999.

Mathie, R. A. and Mann, I. R.: A correlation between extended interals of ULF wave power and storm-time geosynchronoous relativistic electron flux enhancements, Geophys. Res. Lett., 27, 3261, 2000.

Mathie, R. A. and Mann, I. R.: On the solar wind control of Pc5 ULF pulsation power at mid-latitudes: Implications for $\mathrm{MeV}$ electron acceleration in the outer radiation belt, J. Geophys. Res., 106, $29783,2001$.

Meredith, N. P., Horne, R. B., and Anderson, R. R.: Substorm dependence of chorus amplitudes: implications for the acceleration of electrons to relativistic energies, J. Geophys. Res. 106, 13 165, 2001.

Meredith, N. P., Horne, R. B., Iles, R. H. A., Thorne, R. M., Heynderickx, D., and Anderson, R. R.: Outer zone relativistic electron acceleration associated with substorm enhanced whistler mode chorus, J. Geophys. Res., in press, 2002a.

Meredith, N. P., Horne, R. B., Summers, D., Thorne, R. M., Iles, R. H. A., Heynderickx, D., and Anderson, R. R.: Evidence for acceleration of outer zone electrons to relativistic energies by whistler mode chorus, Ann. Geophysicae, this issue, 2002b.

Obara, T., Nagatsuma, T., Den, M., Miyoshi, Y., and Morioka, A.: Main phase creation of "seed" electrons in the outer radiation belt, Earth Planets Space, 52, 41, 2000.

O'Brien, T. P. and McPherron, R. L.: Forecasting the ring current index $D_{s t}$ in real time, J. Atmos. Sol.-Terr. Phy., 62 (14), 1295 2000.

Papetheodorou, G., Woodliffe, R., Johnstone, A. D., Rodgers, D. J., Walton, D. M., and Sims, A. J.: A miniature retarding potential plasma analyser with a differential energy response, in Measurement techniques in Space Plasma Physics: Particles, Geophys, Monog. Ser., 102, 287-293, 1998.

Paulikas, G. A. and Blake, J. B.: Effects of the solar wind on magnetospheric dynamics: Energetic electrons at the synchronous orbit, in: Quantitive modelling of Magnetospheric Processes, (Ed) Olson, W. P., Geophys. Monograph. Series, Vol. 21, pp. 180, AGU, Washington D. C., 1979.

Reeves, G. D.: Relativistic electrons and magnetic storms: 1992 1995, Geophys. Res. Lett., 25, 1 817, 1998.

Summers, D., Thorne, R. M., and Xiao, F.: Relativistic theory of wave-particle resonant diffusion with application to electron acceleration in the magnetosphere, J. Geophys. Res., 103, 20487 , 1998.

Summers, D. and Ma, C.: A model for generating relativistic electron in the Earth's inner magnetosphere based on gyroresonant wave-particle interactions, J. Geophys. Res., 105, 2625, 2000.

Tsurutani, B. T. and Smith, E. J.: Two types of magnetospheric 
ELF chorus and their substorm dependencies, J. Geophys. Res., 82, 5112, 1977.

Tverskaya, L. V.: The latitude position dependence of the relativistic electron maximum as a function of $D_{s t}$, Adv. Space Res., 18, 135, 1996.
Williams, D. J. and Smith, A. M.: Daytime trapped electron intensities at high latitudes at $1100 \mathrm{~km}$, J. Geophys. Res., 70, 541, 1965

Williams, D. J.: A 27-day periodicity in outer zone trapped electron intensities, J. Geophys. Res., 71, 1815, 1966. 